\title{
Design of realistic switches for coupling superconducting solid-state qubits
}

\author{
Markus J. Storct* and Frank K. Wilhelm \\ Sektion Physik and CeNS, Ludwig-Maximilians-Universität, \\ Theresienstr. 37, 80333 München, Germany
}

\begin{abstract}
Superconducting flux qubits are a promising candidate for solid-state quantum computation. One of the reasons is that implementing a controlled coupling between the qubits appears to be relatively easy, if one uses tunable Josephson junctions. We evaluate possible coupling strengths and show, how much extra decoherence is induced by the subgap conductance of a tunable junction. In the light of these results, we evaluate several options of using intrinsically shunted junctions and show that based on available technology, Josephson field effect transistors and high- $\mathrm{T}_{c}$ junctions used as $\pi$-shifters would be a good option, whereas the use of magnetic junctions as $\pi$-shifters severely limits quantum coherence.

PACS numbers: 74.50.+r, 85.25.-j, 03.67.Lx, 03.65.Yz
\end{abstract}

*Electronic address: storcz@theorie.physik.uni-muenchen.de 
Quantum computation promises qualitative improvement of computational power as compared to today's classical computers. An important candidate for the implementation of a scalable quantum computer are superconducting qubits [1, 2]. After experimental demonstration of basic features e.g. in flux qubits [3, 4], the improvement of the properties of such setups involves engineering of couplings and decoherence, see e.g. [5].

To perform universal quantum computation with a system of coupled qubits it is very much desirable to be able to switch the couplings (although there are in principle workarounds [6]). It has already been described that for flux qubits, this can be achieved by using a superconducting flux transformer interrupted by a tunable Josephson junction [2], i.e. a superconducting switch, as shown in Fig. 1] The primary and most straightforward proposal for the implementation of this switch is to use an unshunted DC-SQUID based on tunnel junctions utilizing the same technology as for the qubit junctions. Although this holds the promise of inducing very little extra decoherence, it suffers from two practical restrictions: i) the SQUID loop has to be biased by exactly half a flux quantum in the off-state and ii) the external control parameter is a magnetic flux, which introduces the possibility of flux cross-talk between the qubits and the switch. The combination of i) and ii) implies that even small flux cross-talk will severely perturb the off-state of the switch.

This can be avoided by using different switches: A voltage-controlled device such as a Josephson Field Effect Transistor (JoFET) [7] or an SNS-Transistor completely avoids the cross-talk problem. As an intermediate step [8], one can improve the SQUID by using a large $\pi$-Junction, in order to fix the off-state at zero field. Such $\pi$-junctions can be found in high- $\mathrm{T}_{c}$ systems [9] or in systems with a magnetic barrier [10]. All these junctions are damped by a large subgap conductance because they contain a large number of low-energy quasiparticles.

In this letter, we quantitatively evaluate the coupling strength between two qubits coupled by a switchable flux transformer. We evaluate the strength of the decoherence induced by the subgap current modeled in terms of the RSJ model. Based on this result, we assess available technologies for the implementation of the switch.

We start by calculating the strength $K$ of the coupling between the two qubits without a switch and then show how it is modified by the presence of the switch. From Fig. 1 and the law of magnetic induction we find the following equations for the flux through qubit 1 
and 2 induced by currents in the qubits and the flux transformer

$$
\delta\left(\begin{array}{c}
\Phi_{S} \\
\Phi_{1} \\
\Phi_{2}
\end{array}\right)=\left(\begin{array}{ccc}
M_{T T} & M_{T Q} & M_{T Q} \\
M_{T Q} & M_{Q Q} & 0 \\
M_{T Q} & 0 & M_{Q Q}
\end{array}\right)\left(\begin{array}{c}
I_{S} \\
I_{1} \\
I_{2}
\end{array}\right)
$$

where $M_{Q Q}$ is the self-inductance of the qubits (assumed to be identical), $M_{T Q}$ is the mutual inductance between the transformer and the qubits and the mutual inductance between the qubits is assumed to be negligible. The fluxes $\delta \Phi$ in Eq. (11) are the screening fluxes in the transformer and the two qubits, i.e. the deviations from the externally applied values. Henceforth, we abbreviate Eq. (11) as $\delta \vec{\Phi}=\mathbf{M} \vec{I}$. These formulas are general and can be applied for any flux through the transformer loop. It is most desirable to couple zero net flux through the device, which can be achieved by using a gradiometer configuration [11]. For this gradiometer case, we get $I_{S}=-\left(M_{T Q} / M_{T T}\right)\left(I_{1}+I_{2}\right)$, which we might insert into (11) and find for the inductive energy

$$
E_{\text {ind }}=\left(M_{Q Q}-\frac{M_{T Q}^{2}}{M_{T T}}\right)\left(I_{1}^{2}+I_{2}^{2}\right)-2 \frac{M_{T Q}^{2}}{M_{T T}} I_{1} I_{2} .
$$

The terms resulting from the off-diagonal elements of (11) can directly be identified as the interqubit coupling strength $K=-2\left(M_{T Q}^{2} / M_{T T}\right) I_{1} I_{2}$ which enters the $\hat{\sigma}_{z} \otimes \hat{\sigma}_{z}$ Ising-coupling described in Refs. [2, 12]. Note, that the dynamics of the qubit flux is dominated by the Josephson energies [2], to which the diagonal term is only a minor correction.

We now introduce the tunable Josephson junction into the loop. Using fluxoid quantization, we rewrite the Josephson relation [11] $I_{S}=I_{c} \sin \left(-2 \pi\left(\Phi_{S} / \Phi_{0}\right)\right)$ and insert it into eq. (11). The resulting nonlinear equation can be solved in the following cases: i) If $\left|I_{S} / I_{c}\right| \ll 1$ ("on" state of the switch) we find $K=-2\left(M_{T Q}^{2} / M_{T T}^{\star}\right) I_{1} I_{2}$ with $M_{T T}^{\star}:=M_{T T}+\left(\Phi_{0} / 2 \pi I_{c}\right)=$ $M_{T T}+L_{\text {kin }}(0)$. This can be understood as an effective increase of the self-inductance of the loop by the kinetic inductance of the Josephson junction at zero bias. ii) In the case $\left|I_{S} / I_{c}\right| \approx 1$, "off" state, the circulating current is close to the critical current of the switch, hence the phase drop is $\pm \pi / 2$ and we find an analogous form $K=-2\left(M_{T Q}^{2} / M_{T T}^{\prime}\right) I_{1} I_{2}$ with $M_{T T}^{\prime}=M_{T T}+\left(\Phi_{0} / 4\left|I_{c}\right|\right)$, i.e. at low $I_{c}$ the coupling can be arbitrarily weak due to the enormous kinetic inductance of the junction close to the critical current.

We now turn to the discussion of the decoherence induced by the subgap conductance of the tunable junction. The decoherence occurs due to the flux noise generated through the 
current noise from the quasiparticle shunt. Hence, both qubits experience the same level of noise. The decoherence of such a setup has been extensively studied in Ref. [12] as a function of the environment parameters. In this letter, we evaluate these environment parameters for our specific setup.

We model the junction by the RSJ-model for a sound quantitative estimate of the time scales even though the physics of the subgap conductance is usually by far more subtle than that. We evaluate the fluctuations of the current between two points of the flux transformer loop sketched in figure 1, $L$ is the geometric inductance of the loop, $L_{J}$ is the Josephson inductance characterizing the Josephson contact and $R$ is the shunt resistance. The correlation is given by the fluctuation-dissipation theorem $\langle\delta I \delta I\rangle_{\omega}=\operatorname{coth}(\beta \hbar \omega / 2) \hbar \omega \operatorname{Re} Y(\omega)$, where $Y(\omega)$ is the admittance of the effective circuit depicted in Fig. 2. Following the lines of Ref. [5], this translates into a spectral function of the energy fluctuations of the qubit of the shape $\langle\delta \epsilon(t) \delta \epsilon(0)\rangle_{\omega}=J(\omega) \operatorname{coth}\left(\hbar \omega / 2 k_{B} T\right)$ with $J(\omega)=\alpha \omega^{2} /\left(\omega^{2}+\omega_{c}^{2}\right)$ with the important result that the dimensionless dissipation parameter here reads

$$
\alpha=\frac{4 I_{\text {circ }}^{2} M_{T Q}^{2} L_{J}^{2}}{h R\left(L+L_{J}\right)^{2}}
$$

and a cutoff $\omega_{c}=R\left(L+L_{J}\right) / L L_{J}$. Here, $L_{J}=\Phi_{0} / 2 \pi I_{c}$ is the kinetic inductance of the junction. From (3) we receive in the limit $L \gg L_{J}$ the expression $\alpha \propto 1 / R I_{c}^{2}$ and for $L \approx L_{J}$, $L \ll L_{J}$ it follows that $\alpha \propto 1 / R$. From the results of Ref. [12], we can conclude that $\alpha \approx 10^{-6}$ poses an upper bound for gate operations to be compatible with quantum error correction. In the following sections we will evaluate $\alpha$ for different types of junctions in the switch, a JoFET, an SFS junction and a high- $T_{c}$ junction by inserting typical parameters. We use the normal resistance $R_{N}$ to estimate the shunt resistance in the RSJ model. Here, it is important to note that the parameters $I_{c}$ and $R_{N}$ of the junction determine the suitability of the device as a (low-noise) switch, which are given by a combination of material and geometry properties. In the following we exemplify the calculation of the dissipative effects with several experimental parameter sets.

For present day qubit technology [13] we can assume $L \approx 1 \mathrm{nH}, I_{\text {circ }} \approx 100 \mathrm{nA} M_{T Q} \approx 100$ $\mathrm{pH}$. In the following, we estimate $\alpha$ for a number of junction realizations, adjusting the junction area for sufficient critical current.

A Josephson field-effect transistor (JoFET) can be understood as an SNS junction where the role of the normal metal is played by a doped semiconductor. By applying a gate voltage, 
it is possible to tune the electron density of the semiconductor.

The critical current of such a junction containing $N_{\text {ch }}$ channels can be found using the formula of Kulik and Omel'yanchuk $I_{c}=(\pi \Delta) /\left(R_{N} e\right)$ [11, 14]. $R_{N}=h /\left(2 e^{2} N_{\mathrm{ch}}\right)$ is the point-contact resistance. In a JoFET, the back gate essentially controls $N_{\text {ch }}$. The typical normal resistance is around $R_{N} \approx 10 \Omega$. For a JoFET the critical current of the Josephson junction is $I_{c} \approx 30 \mu \mathrm{A}$ and the Josephson inductance is $L_{J} \approx 11 \mathrm{pH}[7]$.

Inserting the above estimates we get $\alpha \approx 7 \cdot 10^{-6}$. This means that the dissipative effects are weak and a JoFET should be a reasonable switch that poses no new constraints. Besides the obvious technological challenge [7], one drawback of JoFETs is that due to wide junctions with dimensions of around $w=500 \mathrm{~nm}$ they are likely to trap vortices, which can cause $1 / \mathrm{f}$ noise by hopping between different pinning sites. However, this can be reduced by pinning e.g. by perforating the junction.

If we go away from the "on" state with the JoFET, we reduce both $I_{c}$ and $G_{N}$ linearily by depleting the density of states. Fig. 3 shows that we find that the dissipative effects are strongest during the switching process when $L_{J}\left(\rho_{e} / \rho_{e}^{o n}\right) \approx L_{J, 0}$, and not in the "on" state of the switch. In the "off" state of the switch (for $\rho_{e}(0) \rightarrow 0$ ) also $\alpha$ goes to zero. If the switch is tuned from the "off" state to the "on" state, $\alpha$ reaches a local maximum and then decreases again. This makes the JoFET a very attractive switch: It induces an acceptably low level decoherence in the "on" state and can be made completely silent in the "off" state.

An SFS junction in the $\pi$-state is based on a metallic material, thus the estimate of the shunt resistance in the RSJ model yields a much smaller result than in the case of the JoFET, $R \approx 10^{-5} \Omega[10]$. The critical current of the SFS junction is $I_{c} \approx 0.2 \mathrm{~mA}$. Thus, leaving the transformer properties unchanged, we find $L_{J} \approx 1.7 \mathrm{pH}$. Using these estimates the strength of the dissipative effects is of the order of $\alpha \approx 0.16$. This makes such a device unsuitable at the present level of technology, however, it appears that SIFS junctions [15] are by far closer to the desired values, see Fig. 4 ,

High- $T_{c}$ junctions can be realized in different ways. Here, we take from Ref. [9] parameters for a typical noble metal $(\mathrm{Au})$-bridge junction with a film thickness of about $w \approx 100 \mathrm{~nm}$. The product $I_{c} R_{N} \approx 1 \mathrm{mV}$ and $\rho_{N}=8.3 \Omega \mathrm{nm}$. We assume that in principle $I_{c}$ for the $\pi$-state and the 0 -state are the same. For a contact area of around $30 \mathrm{x} 30 \mathrm{~nm}^{2}, I_{c} \approx 1$ $\mathrm{mA}$ and $R_{N} \approx 1 \Omega$. Now the strength of the dissipative effects is easily evaluated to be $\alpha \approx 6.5 \cdot 10^{-8}$, which is way better than SFS $\pi$-junctions and even better than the JoFET. 
We estimated the strength of the dissipative effects that will occur due to the switch for several possible switches. These results are summarized in figure 4 for typical parameters of the analyzed systems. We find that the noise properties of a JoFET and $\pi$-shifters based on High- $\mathrm{T}_{c}$-materials introduce no important noise source. On the other hand, the parameters found from $\pi$-shifters based on magnetic materials are much less encouraging.

We would like to thank T.P. Orlando, P. Baars and A. Marx for useful discussions. Work supported by ARO through Contract-No. P-43385-PH-QC.

[1] Yu. Makhlin, G. Schön, and A. Shnirman, Rev. Mod. Phys. 73, 357-400 (2001).

[2] T.P. Orlando, J.E. Mooij, C.H. van der Wal, L. Levitov, S. Lloyd, J.J. Mazo, Phys. Rev. B 60, 15398 (1999); J.E. Mooij, T.P. Orlando, L. Levitov, L. Tian, C.H. van der Wal, S. Lloyd, Science 285, 1036 (1999).

[3] C.H. van der Wal, A.C.J. ter Haar, F.K. Wilhelm, R.N. Schouten, C.J.P.M Harmans, T.P. Orlando, S. Lloyd, J.E. Mooij, Science 290, 773 (2000).

[4] I. Chiorescu, Y. Nakamura, C.J.P.M. Harmans, and J.E. Mooij, Science 299, 1869 (2003).

[5] C.H. van der Wal, F.K. Wilhelm, C.J.P.M. Harmans, and J.E. Mooij, Eur. Phys. J. B 31, 111 (2003).

[6] X. Zhou, Z.-W. Zhou, G.-C. Guo, and M.J. Feldman, Phys. Rev. Lett 89, 197903 (2002)

[7] A. Richter, Adv. Sol. St. Phys. Vol. 40, 321.

[8] G. Blatter, V.B. Geshkenbein, L.B. Ioffe, Phys. Rev. B 63, 174511 (2001).

[9] K.A. Delin and A.W. Kleinsasser, Supercond. Sci. Technol. 9, 227 (1996).

[10] V.V. Ryazanov, V.A. Oboznov, A.Yu. Rusanov, A.V. Veretennikov, A.A. Golubov, and J. Aarts, Phys. Rev. Lett. 86, 2427 (2001).

[11] M. Tinkham: Introduction to Superconductivity, 2nd ed., McGraw-Hill (1996).

[12] M.J. Storcz and F.K. Wilhelm, Phys. Rev. A 67042319 (2003).

[13] B. Singh and T.P. Orlando, private communication.

[14] I.O. Kulik and A.N. Omelyanchuk, Sov. J. Low Temp. Phys. 3, 459 (1977)

[15] T. Kontos, M. Aprili, J. Lesueur, F. Genêt, B. Stephanidis, and R. Boursier, Phys. Rev. Lett. 89137007 (2002). 


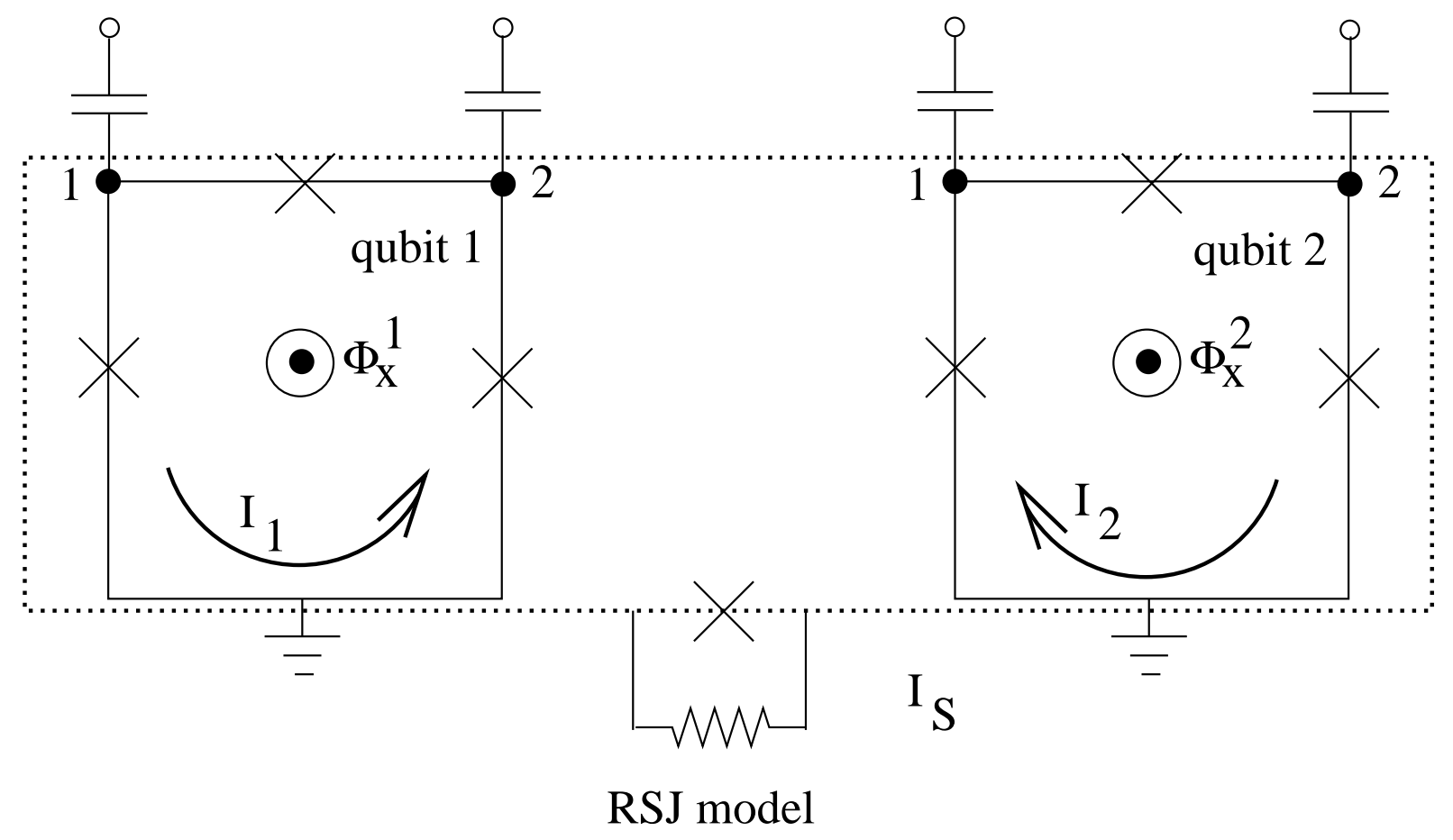

FIG. 1: The flux transformer inductively couples two flux qubits [2]. It can be switched, e.g. by a DC-SQUID or by a tunable shunted Josephson junction.

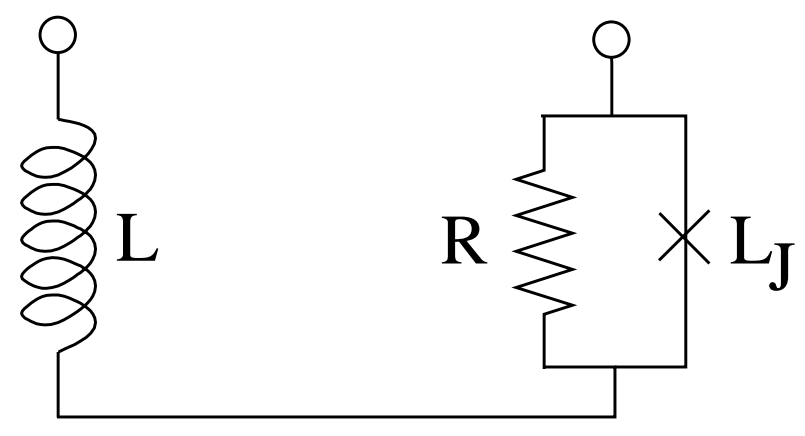

FIG. 2: Equivalent circuit diagram of the flux transformer circuit. The JoFET is modeled by a resistively shunted Josephson junction. 


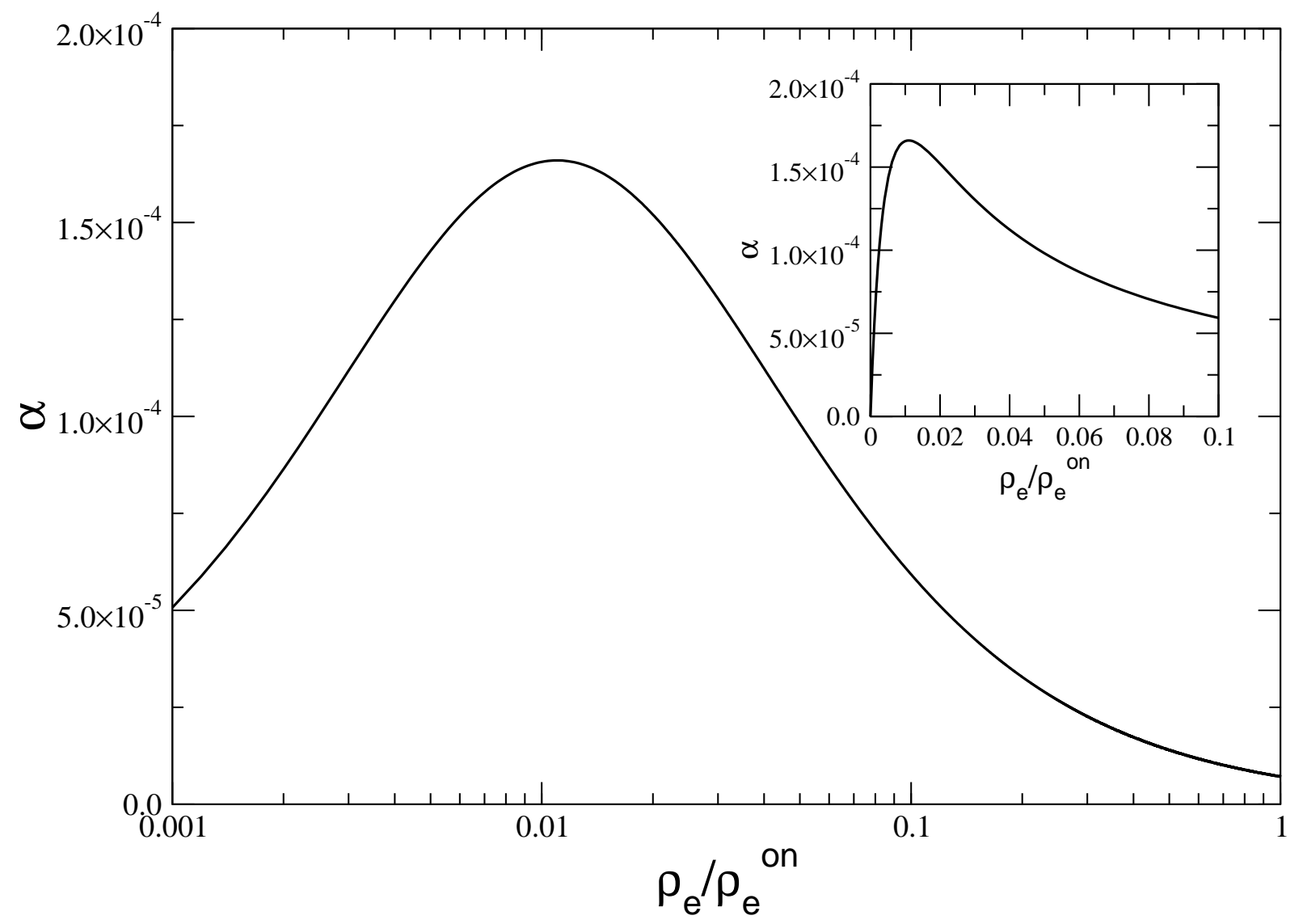

FIG. 3: The dimensionless dissipation parameter $\alpha$ as a function of the electron density in the 2DEG for a JoFET. The inset shows a linear plot of the region with the largest $\alpha$. 


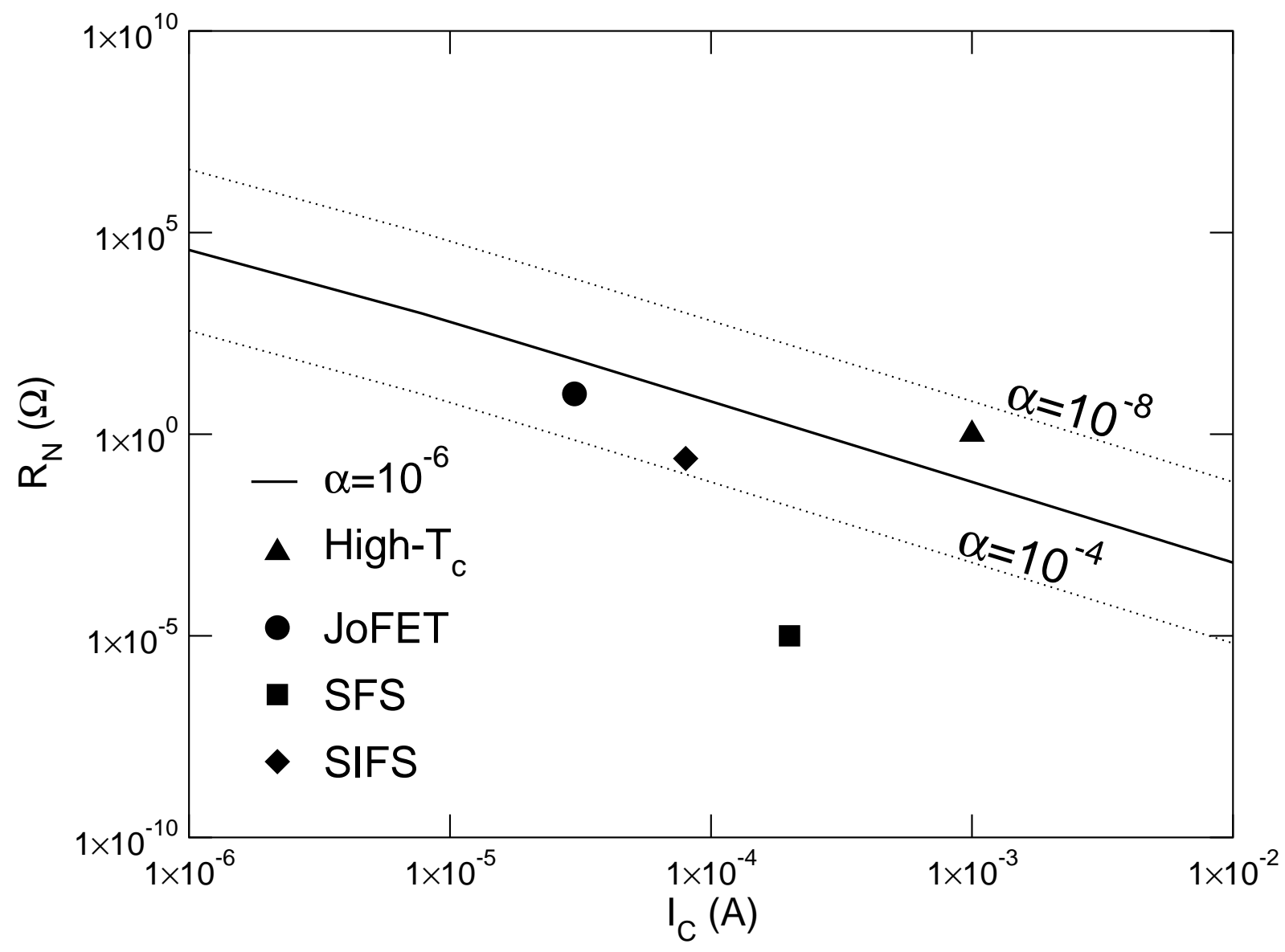

FIG. 4: Log-log plot of normal state resistance versus the critical current of the junction. Here $R_{N}$ is taken as an estimate for the shunt resistance of the junction. The solid line denotes $\alpha=10^{-6}$ and the two dotted lines are for $\alpha=10^{-4}$ (lower line) and $\alpha=10^{-8}$ (upper line). Parameters for the SIFS-junction are $I_{c} \approx 8.5 \cdot 10^{-5} \mathrm{~A}$ and $R_{N} \approx 250 \mathrm{~m} \Omega[15]$. 